\title{
Extensions of Cut-and-Choose Fair Division
}

\author{
Jack M. Robertson and William A. Webb
}

Jack Robertson received his PhD from the University of Utah in 1964 and has been at Washington State University since then. He has broad mathematical interests including combinatorial geometry, graph theory, and mathematics education. He is an avid outdoorsman enjoying fishing, backpacking, golf, gardening, and rockhounding.

Bill Webb received his PhD from Pennsylvania State University in 1968. Originally - and still - a number theorist, he has branched out to work in fair division, combinatorics and cryptography. He enjoys playing tennis, lapidary, square and round dancing, playing classical guitar and is trying his hand at designing glass art.

In its simplest setting the fair division problem asks that two people "fairly" divide a piece of cake. A widely known solution is: "one cuts the other chooses." The general problem is, like most cakes over which it is posed, very rich and has blossomed since its introduction by Steinhaus in 1948 [9]. In the case of $n$ persons, there are various ways to guarantee all persons at least $1 / n$th of the cake by their own assessments $[4,5$, $8,9,10]$. If a piece can be found on which two persons disagree, all can be guaranteed strictly more than $1 / n$th of the cake by their assessment [13]. It is also known how to guarantee each of three persons an "envy free" piece (i.e. nobody thinks another has a preferred piece) using at most five cuts [12]. Existence proofs for envy free portions have been given $[1,3,12]$ and there are two procedures that generate envy-free pieces for $n$ persons, but neither procedure has a bound (as a function of $n$ ) for the number of cuts that may be required [2,7]. Existence proofs have been given to show there are $n$ pieces

Ob man einen Kuchen teilt, eine Erbschaft oder das Volkseinkommen - wenn die Betroffenen die Teilung nicht als fair empfinden, so sind Konflikte vorprogrammiert. Bei der Teilung eines Kuchens unter zwei Parteien können alle Eltern glücklicherweise von der bekannten und eleganten Lösung profitieren: Der eine teilt, der andere wählt! Wen überrascht es, dass sich die Mathematik für diese brilliante Idee weiter interessiert? In der Tat lässt sich das Verfahren auf kompliziertere Situationen verallgemeinern, auf Teilungsprobleme zwischen mehreren Parteien und auf solche mit zusätzlichen, einschränkenden Bedingungen. Wie immer, wenn die Mathematik ein Problem aufgreift, taucht eine Menge neuer und interessanter Fragen auf. Einigen davon gehen Jack Robertson und Bill Webb im vorliegenden Beitrag nach. ust 
which all $n$ players think are equal [1]. "Moving knife" continuous algorithms have been given for the latter problem for $n=2$ [7], for the envy free problem for $n=3$ [11], and the original problem above for any $n[10,12]$. Players can be guaranteed fair rational unequal portions if that is what they deserve [6], and much attention has been given to trying to minimize the number of cuts used to accomplish the various tasks.

The purpose of this note, after all this activity, is to return to the most basic procedure of two person "cut-and-choose" and explore what can be accomplished with a sequence of such steps. In keeping with the simplicity of the cut-and-choose procedure, we will not give the careful mathematical formulation (found widely in print, e.g. [12]) but rather rely on the intuitive aspects of the procedures. Note that the cut-and-choose procedure assumes only that the cutter can cut one piece into two pieces on which the cutter has no preference, that the chooser can exercise a preference on the two pieces presented, and that the total value of the cake or its pieces is not diminished or enhanced by the cut. Thus, a notable feature of such a simple cut-and-choose procedure is that it requires only a preference ranking and not a numerical evaluation of the pieces.

What divisions can be done utilizing only cut-and-choose procedures? When can fair division be accomplished with a finite number of steps? The following discussion provides the answers to these questions.

Looking first at a specific example, suppose $P_{1}$ cuts the cake and $P_{2}$ chooses one of the pieces. Now $P_{2}$ cuts the unchosen piece and $P_{1}$ chooses between the resulting two pieces. Finally $P_{1}$ cuts the new unchosen piece, $P_{2}$ chooses one piece and $P_{1}$ gets what is left. We claim that this procedure quarantees $P_{1}$ at least $3 / 8$ and $P_{2}$ at least $5 / 8$ of the cake. (See Example 1 below.) How do we know this and how do we decide who should cut next? When should we stop? It turns out that the rule is quite easy, works for rational or irrational ratios, and is given in general by:

Procedure I: A Sequence of Cut-and-Choose Steps that Guarantees Two Players $P_{1}$ and $P_{2}$ Fair Pieces in the Ratio $\alpha: \beta$ for any Real Numbers $\alpha>0, \beta>0, \alpha+\beta=1$.

1. Write $\alpha=._{1} \alpha_{2} \alpha_{3} \cdots$ and $\beta=\beta_{1} \beta_{2} \beta_{3} \cdots$ in binary form, using the terminating forms if possible.

2. At the ith stage, $P_{1}$ cuts if $\alpha_{i}=0 ; \quad P_{2}$ cuts if $\beta_{i}=0$; either cuts if $\alpha_{i}=\beta_{i}=1$. The other chooses and keeps (or banks) the chosen piece.

3. The procedure stops in the case $\alpha_{i}=\beta_{i}=1$ with the unchosen piece given to the cutter. Otherwise the procedure continues on the unchosen piece.

Example 1. Players $P_{1}$ and $P_{2}$ are to share the cake in the ratio $3: 5$ or $3 / 8: 5 / 8$.

1. $3 / 8=.011 \quad 5 / 8=.101$

2. a. $P_{1}$ cuts the cake $X=X_{11} \cup X_{12} ; \quad P_{2}$ chooses (wlog) $X_{12}$.

b. $P_{2}$ cuts $X_{11}=X_{21} \cup X_{22} ; \quad P_{1}$ chooses (wlog) $X_{21}$.

c. Either, say $P_{1}$, cuts $X_{22}=X_{31} \cup X_{32} ; \quad P_{2}$ chooses (wlog) $X_{32}$ and $X_{31}$ is given to $P_{1}$.

3. The procedure stops since $\alpha_{3}=\beta_{3}=1$. Player $P_{1}$ has $X_{21} \cup X_{31}$ while $P_{2}$ has $X_{12} \cup X_{32}$. 
If the binary forms are non-terminating the procedure is countably infinite. Since $\alpha+\beta=$ 1 we know $\alpha_{i}+\beta_{i}=1$ unless $\alpha_{i}=\beta_{i}=1$ in the last place of terminating forms.

We must show $P_{1}$ and $P_{2}$ receive pieces they value at least $\alpha$ and $\beta$ respectively. Let $X_{j 1}$ and $X_{j 2}$ be the two pieces produced by the $j$ th cut and let $\mu_{1}\left(X_{j k}\right)$ be the fraction of the entire cake $X$ that piece $X_{j k}$ represents according to player $P_{1} ; \mu_{2}\left(X_{j k}\right)$ is defined similarly for $P_{2}$.

If $X=X_{1} \cup X_{2}$, where $X_{1}$ and $X_{2}$ are the totality of all pieces assigned to $P_{1}$ and $P_{2}$ respectively, we must show $\mu_{1}\left(X_{1}\right) \geq \alpha$ and $\mu_{2}\left(X_{2}\right) \geq \beta$. This will be established by induction. After $k$ steps of Procedure I, $P_{1}$ and $P_{2}$ will have received certain portions of the cake. We will denote these two total banked holdings through step $k$ by $Y_{k}$ and $Z_{k}$ respectively and the remaining unchosen piece by $R_{k}$. Also set $A_{k}=._{1} \alpha_{2} \cdots \alpha_{k}$ and $B_{k}=. \beta_{1} \beta_{2} \cdots \beta_{k}$. We show through $k$ steps $P_{1}$ and $P_{2}$ both think they are doing fine so far and the other is not running ahead.

Claim: For $k=1,2,3 \cdots$

a. $\mu_{1}\left(Y_{k}\right) \geq A_{k}, \quad$ b. $\mu_{1}\left(Z_{k}\right) \leq B_{k}$, c. $\mu_{2}\left(Y_{k}\right) \leq A_{k}$,

d. $\mu_{2}\left(Z_{k}\right) \geq B_{k}$, and $\quad$ e. $\mu_{i}\left(R_{k}\right) \leq \frac{1}{2^{k}}, \quad i=1,2$.

Proof. For $k=1$, the chooser should think he or she receives at least half of the cake while the cutter should not think more than half has been given away. That is exactly what cut-and-choose accomplishes. Inequality (e) is clear for $k=1$ also.

Thus assuming the $k$ th case let us examine what happens at the $(k+1)$ st step.

Case 1: $\alpha_{k+1}=0, \beta_{k+1}=1$.

Player $P_{1}$ will cut $R_{k}$ in two and $P_{2}$ will choose and bank one of the two pieces. We know $\mu_{i}\left(R_{k}\right)=1-\mu_{i}\left(Y_{k}\right)-\mu_{i}\left(Z_{k}\right)$ for $i=1,2$. Since $Y_{k}=Y_{k+1}, \quad \mu_{1}\left(Y_{k+1}\right)=\mu_{1}\left(Y_{k}\right) \geq$ $A_{k}=A_{k+1}$ from the induction assumption (a), and $\mu_{2}\left(Y_{k+1}\right)=\mu_{2}\left(Y_{k}\right) \leq A_{k}=A_{k+1}$ from (c).

Also $\mu_{2}\left(Z_{k+1}\right) \geq \mu_{2}\left(Z_{k}\right)+\frac{1}{2}\left(1-\mu_{2}\left(Y_{k}\right)-\mu_{2}\left(Z_{k}\right)\right)=\frac{1}{2}\left(1+\mu_{2}\left(Z_{k}\right)-\mu_{2}\left(Y_{k}\right)\right) \geq$ $\frac{1}{2}\left(1+B_{k}-A_{k}\right)=\frac{1}{2}+B_{k}-\frac{1}{2}\left(B_{k}+A_{k}\right)=\frac{1}{2}+B_{k}-\frac{1}{2}\left(1-\frac{1}{2^{k}}\right)=B_{k}+\frac{1}{2^{k+1}}=B_{k+1}$. For (b), $\mu_{1}\left(Z_{k+1}\right)=\mu_{1}\left(Z_{k}\right)+\frac{1}{2}\left(1-\mu_{1}\left(Y_{k}\right)-\mu_{1}\left(Z_{k}\right)\right)=\frac{1}{2}\left(1+\mu_{1}\left(Z_{k}\right)-\mu_{1}\left(Y_{k}\right)\right) \leq \frac{1}{2}\left(1+B_{k}-\right.$ $\left.A_{k}\right)=B_{k+1}$, as was seen above. Finally for $(\mathrm{e}), \mu_{i}\left(R_{k+1}\right) \leq \frac{1}{2} \mu_{1}\left(R_{k}\right) \leq \frac{1}{2} \cdot \frac{1}{2^{k}}=\frac{1}{2^{k+1}}$.

Case 2: $\alpha_{k+1}=1, \beta_{k+1}=0$

This is established the same way as Case 1 .

Case 3: $\alpha_{k+1}=\beta_{k+1}=1$.

This case is established by observing that $R_{k+1}$ is empty, $A_{k+1}+B_{k+1}=1$, and by justifying (b) and (d) exactly as in Case 1 . Thus, the claim is established and the procedure accomplishes the required division. 
In fact the procedure just described gives the only sequence of cut-and-choose steps which guarantees pieces in the ratio $\alpha: \beta$. For suppose we have a prescribed sequence of cut-and-choose steps indicating which player is to cut at each step. We will further assume that if the procedure terminates after the $i$ th step that the cutter receives the unchosen piece at that step. (Note that this is equivalent to the $i$ th stage cutter repeatedly choosing on the remainders as the other cuts. We will always opt for the terminating form.)

On the basis of this information about the procedure, let us define

$$
a_{i}=\left\{\begin{array}{l}
0 \text { if } P_{1} \text { cuts and does not receive the unchosen piece at } \\
\text { step } i, \text { or the procedure terminates before step } i . \\
1 \text { if } P_{2} \text { cuts and } P_{1} \text { chooses, or, } P_{1} \text { cuts and } \\
\text { receives the unchosen piece at step } i .
\end{array}\right.
$$

Define $b_{i}$ similarly for $P_{2}$. It is clear that $\left(. a_{1} a_{2} \cdots\right)+\left(. b_{1} b_{2} \cdots\right)=1$ in either the terminating or non-terminating case.

What is the most cake guaranteed $P_{1}$ by this sequence of steps? In particular it may be the case that (i) the two players use the same measure (although they may not realize it) and (ii) $P_{2}$ always cuts halves when $P_{2}$ cuts (maybe unbeknownst to $P_{1}$ ). From the previous discussion above we know this guarantees $P_{2}$ a share $B$ with $\mu_{2}(B) \geq . b_{1} b_{2} \ldots$. Hence, since the players are using the same measure, $\mu_{1}(X-B) \leq 1-. b_{1} b_{2} \cdots=. a_{1} a_{2} \cdots$. So the most this sequence of cut-and-choose steps can guarantee $P_{1}$ in the general case is .$a_{1} a_{2} \cdots$ of the cake. By the same agrument with the players switched, $P_{2}$ is guaranteed in general no more than $b_{1} b_{2} \cdots$ by the procedure. It follows that any sequence of steps other than that found in Procedure I will generate two numbers where either $a_{1} a_{2} \cdots<\alpha$ or $b_{1} b_{2} \cdots<\beta$.

Furthermore, we see that in order to guarantee a fair share for a player, that player must cut halves at each stage. For if the $i$ th stage is the first step where the cutter fails to cut halves, the chooser can take the cutter's larger piece. Again they may be using the same measure. Using the notation in the proof above and assuming $P_{1}$ cut non-halves in step $i$ we then have $\mu_{j}\left(Y_{i-1}\right)=A_{i-1}, \mu_{j}\left(Z_{i-1}\right)=B_{i-1}, \mu_{j}\left(Z_{i}\right)>B_{i}$ and $\mu_{j}\left(R_{i}\right)<1 /\left(2^{i}\right)$ for $j=1,2$. But, arguing as above, (for the share.$\alpha_{i+1} \alpha_{i+2} \cdots$ on $R_{i}$ ) the most $P_{1}$ can then receive is $A_{i-1}+2^{i}\left(\alpha-A_{i-1}\right) \mu_{1}\left(R_{i}\right)<A_{i-1}+\left(\alpha-A_{i-1}\right)=\alpha$.

In summary, Procedure I is the unique sequence of cut-and-choose steps which guarantees the fair shares in the given ratio, and the only strategy which guarantees those fair shares is for the cutter to always cut halves.

Example 2: Suppose $P_{1}, P_{2}$, and $P_{3}$ are to share in the ratios $1 / 2: 1 / 3: 1 / 6$. Using Procedure I, $P_{1}$ and $P_{2}$ can first divide the cake in the ratio $1 / 2: 1 / 3$ which is $3 / 5: 2 / 5$. Then $P_{3}$ can repeat Procedure I in the ratio $1 / 6: 5 / 6$ with each of $P_{1}$ and $P_{2}$. Then $P_{1}$ will have at least $5 / 6 \cdot 3 / 5=1 / 2, P_{2}$ will have at least $5 / 6 \cdot 2 / 5=1 / 3$ and $P_{3}$ will have at least $1 / 6$ th of everything. In order to avoid a sequence of three infinite procedures back to back, a single countable process can be described using a diagonalization procedure that permits $P_{3}$ to start the division of pieces with $P_{1}$ and $P_{2}$ as soon as they are chosen 
by $P_{1}$ or $P_{2}$. Once a piece is banked in either the $P_{1}, P_{3}$ or $P_{2}, P_{3}$ division, it remains banked and uncut.

For $P_{1}, \cdots, P_{n}$ to share in the ratios $\alpha_{1}: \alpha_{2}: \cdots: \alpha_{n}$ first have $P_{1}, \cdots, P_{n-1}$ share in the ratios $\frac{\alpha_{1}}{1-\alpha_{n}}: \frac{\alpha_{2}}{1-\alpha_{n}}: \cdots: \frac{\alpha_{n-1}}{1-\alpha_{n}}$. Then $P_{n}$ will share with each of $P_{1}, \cdots, P_{n-1}$ in the ratio $\alpha_{n}: 1-\alpha_{n}$. The procedures can be diagonalized so that a single countable sequence suffices.

When is a finite procedure possible? For $n=2$ this is an easy question, the procedure is finite if and only if $\alpha /(\alpha+\beta)=a / 2^{m}$ for some positive integers $a$ and $m$. Hence, the ratio $5: 11$ is accomplished by 4 steps while the ratio $1 / \pi:(\pi-1) / \pi$ requires an infinite procedure (even on pies).

The case for three or more players is more interesting. For example, suppose the ratios are 12:3:1. If $P_{1}$ and $P_{2}$ divide first, the ratio is $4 / 5: 1 / 5$ which leads to an infinite process. However, if $P_{2}$ and $P_{3}$ divide first in the ratio $3 / 4: 1 / 4$, that process is finite and must be followed by $P_{1}$ dividing with each of $P_{2}$ and $P_{3}$ in the ratio $3 / 4: 1 / 4$ each of which is finite. In general the inductive procedure given above for $n$ players is finite if and only for some permutation $a_{1}: a_{2}: \cdots: a_{n}$ of the ratios each of the fractions $a_{i} /\left(a_{1}+a_{2}+\cdots+a_{i}\right), 2 \leq i \leq n$, can be written as $a / 2^{m}$.

The example above shows that the order of the divisions can be important. Indeed the procedure cannot be finite for all possible permutations of the ratios. Suppose we have three positive numbers $\alpha, \beta, \gamma$ with $\alpha+\beta+\gamma=1$ so that the overall process is finite regardless which pair goes first. This would require $\alpha /(\alpha+\beta)$ and $\beta /(\alpha+\beta)$ to have the form $a / 2^{m}$ and $b / 2^{m}$ where $a+b=2^{m}$. Thus, $\alpha / \beta=a / b$ is rational as is $\alpha / \gamma$. It follows that we can assume $\alpha: \beta: \gamma$ are the same ratios as $n_{1}: n_{2}: n_{3}$ where all $n_{i}$ are integers. Since we are assuming the divisions in the ratios $n_{1}: n_{2}, n_{1}: n_{3}, n_{2}: n_{3}$ and $\left(n_{1}+n_{2}\right): n_{3}$ are all finite procedures we have:

$$
\begin{array}{ll}
n_{1}+n_{2} & =2^{j} \\
n_{1}+n_{3} & =2^{k} \\
n_{2}+n_{3} & =2^{l} \\
n_{1}+n_{2}+n_{3} & =2^{m}
\end{array}
$$

where $j, k, l \geq 1$ and $m \geq 2$.

Thus $2^{m}=2^{j-1}+2^{k-1}+2^{l-1}$ and this equality requires (wlog) $j-1=m-1, \quad k-1=$ $l-1=m-2$. But then $n_{1}+n_{2}=2^{m}$ and $n_{3}=0$ contradicting $\gamma>0$.

We have seen above that using a sequence of strict cut-and-choose steps, a finite procedure is only possible when the binary forms terminate. We next describe a finite procedure "in the spirit of cut and choose" which applies to all rational ratios $a: b$. As before, each step will have a cutter with the other player choosing and banking one of the two pieces produced at that step. We will have to use more than a simple preference evaluation on pieces however.

In what follows we will assume $a$ and $b$ are positive integers and that the cake $X$ is to be divided in the ratio $a: b$ between $P_{1}$ and $P_{2}$ respectively. We will describe a finite, modified cut-and-choose procedure which we will denote $P(a, b)$. 
The procedure $P(1,1)$ is the original cut-and-choose where each person gets at least $1 / 2$ of the cake. Now assume $P(c, d)$ has been defined for all $c$ and $d$ such that $c+d<n$, so as to assure $P_{1}$ receives at least $c /(c+d)$ and $P_{2}$ receives at least $d /(c+d)$ of the cake in a finite number of steps. With $a+b=n \geq 3$, we will now describe $P(a, b)$. Intuitively, the cutter cuts in the ratio $\alpha: \beta$ where $\alpha+\beta=a+b, \alpha$ and $\beta$ are integers and $\alpha / \beta$ is as close to 1 as possible. Formally:

Procedure II: A Finite Sequence of Modified Cut-and-Choose Steps that Guarantees Two Players, $P_{1}$ and $P_{2}$, Fair Pieces in the Rational Ratio $a: b$.

(1) For $a>0, P(a, 0)$ is $P_{1}$ takes the piece; for $b>0, P(0, b)$ is $P_{2}$ takes the piece and the procedure stops. For $a=b=1, P(a, b)$ is either player cuts halves, the other chooses. The remaining piece goes to the cutter and the procedure stops.

Assume for (2) and (3) that $a b \neq 0, a+b>2$ and $(a, b)=1$.

(2) If $a+b$ is even and (wlog) $a<b$ then $P_{1}$ cuts equal pieces, $X=X_{1} \cup X_{2}$. Then $P_{2}$ chooses the larger of $X_{1}$ or $X_{2}$ and procedure $P\left(a, \frac{b-a}{2}\right)$ is applied to the other piece.

(3) If $a+b$ is odd and (wlog) $a<b$ then $P_{1}$ cuts $X=X_{1} \cup X_{2}$ in the ratio $\frac{a+b-1}{2}$ : $\frac{a+b+1}{2}$ respectively. Player $P_{2}$ chooses $X_{1}$ if it is considered to have value at least $\frac{a+b-1}{2} \cdot \frac{1}{a+b}$ and chooses $X_{2}$ if it is at least $\frac{a+b+1}{2} \cdot \frac{1}{a+b}$. If $P_{2}$ chooses $X_{1}$ apply $P\left(a, \frac{b-a+1}{2}\right)$ to $X_{2}$. If $P_{2}$ chooses $X_{2}$ apply $P\left(a, \frac{b-a-1}{2}\right)$ to $X_{1}$.

Since $a+\frac{1}{2}(b-a+1)=\frac{1}{2}(a+b)+1 / 2<a+b=n$, all of the specified finite number of additional procedures are inductively defined. Also, since the two values against which $X_{1}$ and $X_{2}$ are measured in (3) have sum one, one of the two choices must be satisfactory to $P_{2}$.

We can now verify that $P_{1}$ will get at least $\frac{a}{a+b}$ and $P_{2}$ will get at least $\frac{b}{a+b}$.

For example, suppose $a+b$ is odd and $P_{2}$ chooses $X_{1}$. Then $P_{1}$ is guaranteed at least $a /\left(\frac{b+a+1}{2}\right)$ of $X_{2}$ by procedure $P\left(a, \frac{b-a+1}{2}\right)$ and $P_{1}$ views $X_{2}$ as worth $\frac{a+b+1}{2} \cdot \frac{1}{a+b}$. Thus, $P_{1}$ gets at least $\frac{2 a}{b+a+1} \cdot \frac{a+b+1}{2(a+b)}=\frac{a}{a+b}$.

Similarly, if $a+b$ is odd and $P_{2}$ chooses $X_{2}$ then $P_{1}$ gets at least $\frac{2 a}{b+a-1} \cdot \frac{a+b-1}{2(a+b)}=$ $\frac{a}{a+b}$.

To prove that $P_{2}$ will get at least $\frac{b}{a+b}$ we could give an argument like that for $P_{1}$. Rather let us assume that $P_{2}$ is not satisfied by either choice presented in Case 3. 
Then

$$
\begin{aligned}
& \mu_{2}\left(X_{1}\right)+\left(\frac{b-a+1}{a+b+1}\right) \mu_{2}\left(X_{2}\right)<\frac{b}{a+b} \text { if } P_{2} \text { chooses } X_{1}, \text { and } \\
& \mu_{2}\left(X_{2}\right)+\left(\frac{b-a-1}{a+b-1}\right) \mu_{2}\left(X_{1}\right)<\frac{b}{a+b}, \text { if } P_{2} \text { chooses } X_{2} .
\end{aligned}
$$

Adding these inequalities we obtain $2 b \mu_{2}\left(X_{1}\right)+2 b \mu_{2}\left(X_{2}\right)<\frac{b}{a+b}(2 a+2 b)$, or $\mu_{2}\left(X_{1}\right)+$ $\mu_{2}\left(X_{2}\right)<1$. This contradicts $\mu_{2}\left(X_{1}\right)+\mu_{2}\left(X_{2}\right)=\mu_{2}(X)=1$.

The case for $a+b$ even is similar.

Example 3: Suppose the ratio is $5: 8$ with player $P_{1}$ receiving the smaller portion. The diagram in Fig. 1 summarizes the branching procedure.

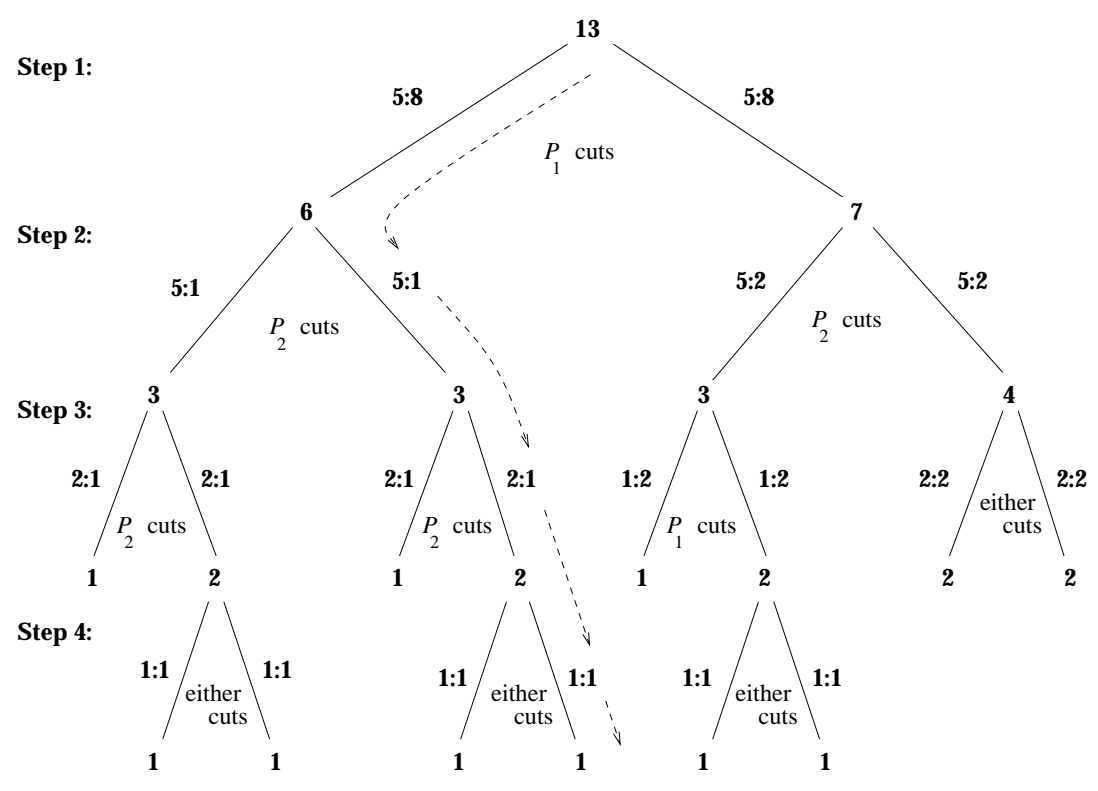

Fig. 1 Branching procedure

For one instance, in the path shown, $P_{1}$ cuts in the ratio $6 / 13: 7 / 13 ; P_{2}$ chooses and banks the $7 / 13$ piece; $P_{2}$ cuts the other piece in the ratio $3 / 6 ; 3 / 6 ; \quad P_{1}$ chooses and banks the left piece; $P_{2}$ cuts in the ratio $2 / 3: 1 / 3 ; P_{1}$ chooses and banks the $1 / 3$ piece; either cuts the remaining piece in halves, the other chooses first with the chosen piece given to the last cutter. 


\section{Bibliography}

[1] N. Alon. Splitting necklaces, Adv. in Math. 63 (1987), 247-253.

[2] S.J. Brams and A.D. Taylor. An envy-free cake division algorithm, Amer. Math. Monthly 102 (1995), 9-18.

[3] L.E. Dubins and E.H. Spanier. How to cut a cake fairly, Amer. Math. Monthly 68 (1961), 1-17.

[4] S. Even and A. Paz. A note on cake cutting, Discrete Appl. Math. 7 (1984), 285-296.

[5] A.M. Fink. A note on the fair division problem, Math. Mag. (1964), 341-342.

[6] K. McAvaney, J. Robertson, W. Webb. Ramsey partitions of integers and fair division, Combinatorica 12, No. 2, (1992), 193-201.

[7] J.M. Robertson and W.A. Webb. Near Exact and Envy Free Cake Division, in press.

[8] T.L. Saaty. Optimization in integers and related extremal problems, McGraw-Hill, New York, (1970).

[9] H. Steinhaus. The problem of fair division, Econometrica 16 (1948), 101-104.

[10] H. Steinhaus. Sur la division pragmatique Econometrica (supplement) 17 (1949), 315-319.

[11] W. Stromquist. How to cut a cake fairly, Amer. Math. Monthly 87 (1980), 640-644.

[12] D.R. Woodall. Dividing a cake fairly, Journal of Math. Anal. Appl. 78 (1980), 233-247.

[13] D.R. Woodall. A note on the cake-division problem, J. Combin. Theory Ser. A 42 (1986), 300-301.

Jack M. Robertson and William A. Webb

Department of Pure and Applied Mathematics

Washington State University

Pullman, WA 99164-3113, USA 